\title{
Fitts' Law Index of Difficulty Evaluated and Extended for Screen Size Variations
}

\author{
Hidehiko Okada and Takayuki Akiba \\ Kyoto Sangyo University \\ Japan
}

\section{Introduction}

It is well-known as Fitts' law that the time for a user to point a target can be modelled as a linear function of "index of difficulty (ID)", where ID is formulated as a function of the target size and distance (Fitts, 1954; MacKenzie, 1992).

$$
\begin{gathered}
\mathrm{t}=\mathrm{a}+\mathrm{b} * \mathrm{ID} \\
\mathrm{ID}=\log _{2}(\mathrm{~A} / \mathrm{W}+1)
\end{gathered}
$$

In Eqs. (1-2), $\mathrm{t}$ is the pointing time, $\mathrm{A}$ is the amplitude (distance) to the target, $\mathrm{W}$ is the target size and $\mathrm{a}, \mathrm{b}$ are constants that depend on experiment conditions. ID is larger as $\mathrm{A}$ is larger and/or $W$ is smaller. Values of $a$ and $b$ in Eq. (1) are determined by sampling (A, W, $t)$ data and applying the linear regression analysis to the data. Eq. (2) shows that ID values are the same for $(\mathrm{A}, \mathrm{W})$ and $(\mathrm{nA}, \mathrm{nW})$ where $\mathrm{n}>0$.

This research is motivated by recent smart phones that employ touch UIs. Compared with other touch screen devices such as tablet PCs, mobile phones have smaller screens so that widgets on mobile phone screens are likely to be smaller. Widgets can be designed for devices with various screen sizes so that theoretical ID values in Eq. (2) are consistent among the devices: larger/smaller sizes \& distances for larger/smaller screens. If ID in Eq. (2) is an appropriate index of actual pointing difficulty independently of screen sizes, users' pointing performances on the same device are consistent among widget designs $(\mathrm{A}, \mathrm{W})$ and (nA, nW): note that $\mathrm{a}, \mathrm{b}$ in Eq. (1) are constant (independent to ID) so that $\mathrm{a}, \mathrm{b}$ must be the same for two data sets sampled with the two widget designs (A, W) and (nA, nW). The aim of this research is to investigate whether the above is true: appropriateness of the ID formulation in Eq. (2) is evaluated from the viewpoint of dependency on screen sizes, by experiments with participants.

Limitations of Fitts' law have been researched and extensions have been proposed. For example, an extension for 2D pointing tasks was proposed (MacKenzie \& Buxton, 1992). Our research aims at investigating possible limitations on screen sizes. A related research was previously reported (Oehl et al., 2007). They investigated how display size influenced pointing performances on a touch UI and reported that in large displays a fast and comparably accurate execution was chosen in contrast to a very inaccurate and timeconsuming style in small displays. In their research the size of small screen was 6.5", and only a large screen touch UI device was utilized for user experiments: screen sizes were 
controlled by means of software program as virtual screens on the device display. In our research, the size of small screen is less than $3^{\prime \prime}$, and a commercial smaller-screen mobile device is utilized.

\section{Experiments}

\subsection{Test tasks}

Participants were asked to point targets on a screen. A test task consisted of pointing two rectangle targets (target 1,2) in a predefined order. An "attempt" was the two successive pointings of target 1 and 2, and a test task consisted of a predefined number set of the attempts. For each combination of experiment conditions, each participant was asked to perform a predefined set of the tasks. The pointing operations were logged for later analyses of pointing speed and accuracy.

\subsection{Conditions}

\subsubsection{Devices}

Three commercial devices were used in our experiment: two tablet PCs and a PDA which have a $\left\{10.2^{\prime \prime}, 6.0^{\prime \prime}, 2.8^{\prime \prime}\right\}$ touch screen respectively. The PDA was selected because several recent smart phones have such small touch screens (i.e., the PDA was used as a substitute for the recent smart phones). Screen sizes of the devices were relatively larger/middle/smaller. In this paper, these devices are denoted as devices L/M/S respectively. Participants performed test tasks by using a stylus attached to each of the three devices ${ }^{1}$.

\subsubsection{Target sizes \& distances}

For each of the three devices, two sets of targets were designed so that ID values in Eq. (2) were consistent between the two sets. Targets in one of the two sets were designed with larger sizes and distances, and those in the other were designed with smaller ones. Specific designs of the two target sets are described later. In this paper, these two target sets are denoted as targets $\mathrm{L} / \mathrm{S}$ respectively.

\subsubsection{Errors}

Pointing speed and accuracy are usually a trade-off (Plamondon \& Alimi, 1997). Participants performed tasks under each of two error conditions: errors acceptable or not. In a test task where errors were acceptable, a participant could continue the task even if s/he made an error (mispointing), and the task was complete when the count of no-error attempts reached to a predefined number. In a condition where errors were not acceptable, a test task was cancelled by an error and the task was retried until the count of no-error attempts reached to a predefined number. The error condition was told to each participant before performing each task: s/ he had to try a task more carefully in the "errors not acceptable" condition.

\footnotetext{
${ }^{1}$ Differences in stylus designs may affect pointing performances (Ren \& Mizobuchi, 2005). It is assumed in our research that the stylus attached to each device is designed optimal for the device so that the stylus contributes to achieve better performances on the device than other styluses.
} 


\subsection{Pointing target designs}

Table 1 shows the design of target sizes and distances. Values for the device $\{M, L\}$ were determined as [values for the device S] * [the ratio of screen sizes, i.e., 6.0/2.8 for the device $\mathrm{M}$ and 10.2/2.8 for the device L]. ID values were designed to range in [2.00, 3.50] consistently among the devices $\{S, M, L\}$ and the targets $\{S, L\}$. The size of target 1 was fixed to $6.0 \mathrm{~mm}$, empirically found to be easy enough to point first, for all conditions. Positions of targets 1 and 2 were randomly determined for each attempt under the following two constraints.

- All areas of both targets were inside the device screen.

- Distance between center points of the two targets was a predefined value.

\begin{tabular}{|c|c|c|c|c|c|c|c|c|c|c|c|c|}
\cline { 2 - 14 } \multicolumn{1}{c|}{} & \multicolumn{4}{c|}{ Device S } & \multicolumn{4}{c|}{ Device M } & \multicolumn{4}{c|}{ Device L } \\
\cline { 2 - 15 } \multicolumn{1}{c|}{} & \multicolumn{3}{c|}{ Targets S } & \multicolumn{2}{c|}{ Targets L } & \multicolumn{2}{c|}{ Targets S } & \multicolumn{2}{c|}{ Targets L } & \multicolumn{2}{c|}{ Targets S } & \multicolumn{2}{c|}{ Targets L } \\
\hline ID & W & A & W & A & W & A & W & A & W & A & W & A \\
\hline 2.00 & 4.00 & 12.00 & 12.00 & 36.00 & 8.53 & 25.60 & 25.60 & 76.80 & 14.61 & 43.82 & 43.82 & 131.45 \\
\hline 2.15 & 3.80 & 13.07 & 11.40 & 39.20 & 8.11 & 27.87 & 24.32 & 83.62 & 13.88 & 47.71 & 41.63 & 143.12 \\
\hline 2.30 & 3.60 & 14.13 & 10.80 & 42.39 & 7.68 & 30.14 & 23.04 & 90.42 & 13.15 & 51.59 & 39.44 & 154.77 \\
\hline 2.45 & 3.40 & 15.18 & 10.20 & 45.53 & 7.25 & 32.38 & 21.76 & 97.14 & 12.41 & 55.42 & 37.24 & 166.26 \\
\hline 2.60 & 3.20 & 16.20 & 9.60 & 48.60 & 6.83 & 34.56 & 20.48 & 103.69 & 11.68 & 59.16 & 35.05 & 177.47 \\
\hline 2.75 & 3.00 & 17.18 & 9.00 & 51.54 & 6.40 & 36.65 & 19.20 & 109.96 & 10.95 & 62.74 & 32.86 & 188.21 \\
\hline 2.90 & 2.80 & 18.10 & 8.40 & 54.30 & 5.97 & 38.61 & 17.92 & 115.84 & 10.22 & 66.09 & 30.67 & 198.27 \\
\hline 3.05 & 2.60 & 18.93 & 7.80 & 56.80 & 5.55 & 40.39 & 16.64 & 121.17 & 9.49 & 69.13 & 28.48 & 207.40 \\
\hline 3.20 & 2.40 & 19.66 & 7.20 & 58.97 & 5.12 & 41.93 & 15.36 & 125.79 & 8.76 & 71.77 & 26.29 & 215.31 \\
\hline 3.35 & 2.20 & 20.23 & 6.60 & 60.70 & 4.69 & 43.16 & 14.08 & 129.49 & 8.03 & 73.88 & 24.10 & 221.63 \\
\hline 3.50 & 2.00 & 20.63 & 6.00 & 61.88 & 4.27 & 44.01 & 12.80 & 132.02 & 7.30 & 75.32 & 21.91 & 225.96 \\
\hline
\end{tabular}

(ID: bits, W\&A: mm)

Table 1. Target sizes and distances

Fig. 1 shows a screenshot of targets 1 and 2 for the device $M$ and the targets L. The targets 1 and 2 are the black and white rectangles respectively (the target colors were consistent for all the devices). The two targets were shown at the same time, and each participant was asked to find both targets before s/he pointed the target 1 . This was because visual search time should not be included in the pointing time interval. After an attempt of pointing targets 1 and 2, new targets were shown for the next attempt.

\subsection{Methods of experiments}

Condition combinations were 12 in total: $\{$ the devices $\mathrm{S}, \mathrm{M}, \mathrm{L}\} *\{$ the targets $\mathrm{S}, \mathrm{L}\} *\{$ errors "acceptable", "not acceptable" $\}$. Each participant was asked to perform four trials of a task under each of the 12 condition combinations.

The number of attempts in a task trial was 11 (of which ID=2.00-3.50 shown in Table 1) for the "errors not acceptable" condition: none of the 11 attempts had to be an error. For the "errors acceptable" condition, a task trial included 11 successful attempts for the 11 IDs respectively in Table 1 and $0+$ error attempts.

Each participant first performed a training task trial under each of the 12 condition combinations (thus, 12 training trials), and then performed tasks in a random order of the 12 condition combinations. The order of the 11 IDs in a trial was also randomized for each trial. 


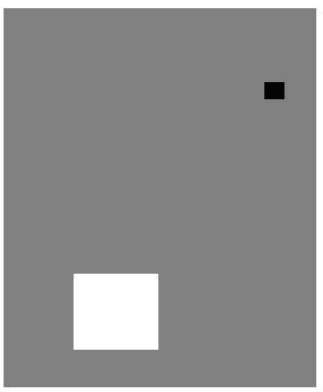

Fig. 1. Screenshot for target pointing tasks

\subsection{Participants}

Twelve subjects participated in the experiment, but 3 of the 12 subjects could for the devices $\mathrm{S}$ and $\mathrm{L}$ only due to the experiment schedule. Thus, users' pointing $\log$ data set $(\mathrm{A}, \mathrm{W}, \mathrm{t})$ were collected with 12 subjects for the devices $S$ and L but 9 subjects for the device $M$. The 12 participants were university graduate or undergraduate students. They were all novices in using devices with touch-by-stylus UIs, but they had no trouble in performing test tasks after the 12 training trials.

\subsection{Logging pointing operations}

The following data was recorded for each pointing (each tap by a stylus) into log files.

- $\quad$ Target: 1 or 2

- Target position: $(x, y)$ values

- Target width and height: pixels

- Tapped position: $(x, y)$ values

- Tap time: msec

- $\quad$ Error: Yes or No

The tapped position and the tap time were logged when the stylus was landed on the screen, and the pointing was judged as an error or not based on the tapped position. No attempt was observed for which the stylus was landed on the target 1 , moved into the target 2 and left off.

\section{Data analyses and findings}

Pointing speed and accuracy were measured by throughput (ISO 9241, 2000) and error rate respectively. In this research, $t$ is the interval from the target 1 tap time to the target 2 tap time, $\mathrm{A}$ is the Euclid distance between the tapped points for targets 1 and 2, and $\mathrm{W}$ is the target width (= height). Throughput is defined as ID/ $t$ in Eqs. (1-2). (ID, $t$ ) could be observed for each attempt, so a throughput value could also be obtained for each attempt. To measure pointing accuracy, error rate was defined.

$$
\text { Error rate }=(\# \text { error attempts in a task trial }) /(\# \text { total attempts in the trial })
$$

Error rate could be calculated for only the condition "errors acceptable" because the data under the condition "errors not acceptable" didn't include any error attempt (if an error was 
occurred in a trial under the condition "errors not acceptable", the trial was cancelled and retried).

Mean and standard deviation (SD) values of the throughput and the error rate were calculated to compare user performances on targets $S$ to those on targets L. Throughput mean and SD values were calculated from the data of $\{\operatorname{tp}(s, t, a)\}$ for all of the subjects, the task trials and the attempts in a task: $\operatorname{tp}(\mathrm{s}, \mathrm{t}, \mathrm{a})$ denotes the throughput value for the $\mathrm{s}$-th subject, $\mathrm{t}$-th task and the a-th attempt in the $\mathrm{t}$-th task by the s-th subject. Error rate mean and SD values were calculated from the data of $\{\operatorname{er}(\mathrm{s}, \mathrm{t})\}$ for all of the subject and the task trials: er $(s, t)$ denotes the error rate value for the s-th subject and the $t$-th task.

In addition, it was tested by t-test whether there was a significant difference between population mean values of throughput and error rate for the two conditions of targets S\&L.

It should be noted that error attempts were included in the data under the condition "errors acceptable". Error attempts might be faster (of larger throughput values) than successful attempts. In the following of this chapter, throughput values were calculated with both of successful and error attempt data.

Table 2 shows mean and SD values of the throughput, and Table 3 shows those of the error rate. Tables $4 \& 5$ show t-test results for throughput and error rate respectively. In Tables $4 \& 5, * *$-marked $\mathrm{t}$-scores are those with $\mathrm{p}<0.01$, and non-marked $\mathrm{t}$-scores are those with $\mathrm{p}>0.05$.

\begin{tabular}{|c|c|c|c|c|c|c|c|}
\cline { 3 - 8 } \multicolumn{2}{c|}{} & \multicolumn{2}{c|}{ Device S } & \multicolumn{2}{c|}{ Device M } & \multicolumn{2}{c|}{ Device L } \\
\cline { 3 - 8 } \multicolumn{2}{c|}{} & Targets S & Targets L & Targets S & Targets L & Targets S & Targets L \\
\hline \multirow{2}{*}{ Acceptable } & Mean & 5.73 & 5.73 & 5.86 & 5.76 & 5.52 & 4.76 \\
\cline { 2 - 8 } & SD & 1.37 & 1.14 & 1.30 & 1.80 & 1.34 & 0.87 \\
\hline \multirow{2}{*}{ Not acceptable } & Mean & 5.15 & 5.57 & 5.69 & 5.63 & 5.32 & 4.60 \\
\cline { 2 - 8 } & SD & 1.20 & 1.21 & 1.30 & 1.78 & 1.23 & 0.97 \\
\hline
\end{tabular}

Table 2. Mean and SD values of throughput (bit/sec)

\begin{tabular}{|c|c|c|c|c|c|c|c|}
\cline { 3 - 8 } \multicolumn{2}{c|}{} & \multicolumn{2}{c|}{ Device S } & \multicolumn{2}{c|}{ Device M } & \multicolumn{2}{c|}{ Device L } \\
\cline { 3 - 8 } \multicolumn{2}{c|}{} & Targets S & Targets L & Targets S & Targets L & Targets S & Targets L \\
\hline \multirow{2}{*}{ Acceptable } & Mean & 11.23 & 0.52 & 0.93 & 0.69 & 1.56 & 0.52 \\
\cline { 2 - 8 } & SD & 10.35 & 2.04 & 2.66 & 2.34 & 3.29 & 2.04 \\
\hline
\end{tabular}

Table 3. Mean and SD values of error rate (\%)

\begin{tabular}{|c|c|c|c|c|c|c|}
\cline { 2 - 7 } \multicolumn{1}{c|}{} & \multicolumn{2}{c|}{ Device S } & \multicolumn{2}{c|}{ Device M } & \multicolumn{2}{c|}{ Device L } \\
\cline { 2 - 7 } & Acceptable & $\begin{array}{c}\text { Not } \\
\text { acceptable }\end{array}$ & Acceptable & $\begin{array}{c}\text { Not } \\
\text { acceptable }\end{array}$ & Acceptable & $\begin{array}{c}\text { Not } \\
\text { acceptable }\end{array}$ \\
\hline Targets S/L & $\mathrm{t}=3.65 * 10^{-3}$ & $\mathrm{t}=-5.74^{* *}$ & $\mathrm{t}=0.875$ & $\mathrm{t}=0.514$ & $\mathrm{t}=11.04^{* *}$ & $\mathrm{t}=10.66^{* *}$ \\
\hline
\end{tabular}

Table 4. T-test for throughput

\begin{tabular}{|c|c|c|c|}
\cline { 2 - 4 } \multicolumn{1}{c|}{} & \multicolumn{3}{c|}{ Acceptable } \\
\cline { 2 - 4 } \multicolumn{1}{c|}{} & Device S & Device M & Device L \\
\hline Targets S/L & $\mathrm{t}=7.03^{* *}$ & $\mathrm{t}=0.393$ & $\mathrm{t}=1.87$ \\
\hline
\end{tabular}

Table 5. T-test for error rate 
These tables revealed the followings.

- On the device L participants could point targets S significantly faster than targets L, but on the devices S\&M they couldn't. Instead, on the device S, they could point targets L significantly faster than targets $S$ under the condition "errors not acceptable". This result indicates that, even though ID values by Eq. (2) are designed consistently among targets S\&L, users' pointing speeds will not be consistent: faster for larger/smaller size\&distance widgets on smaller/larger screen devices, respectively.

- On the devices M\&L no significant difference was observed in the pointing accuracy among targets S\&L, but on the device S participants could point targets L significantly more accurately than targets $S$. This result indicates that, even though ID values by Eq. (2) are designed consistently among targets S\&L, users' pointing accuracies will not be consistent too: more accurate for larger size\&distance widgets on smaller screen devices.

Thus, it is found that the ID definition in Eq. (2) may not consistently capture actual pointing difficulty among target designs. The result of our experiment shows that, on a smaller/larger screen, targets with smaller/larger sizes\&distances are actually more difficult to point than those with larger/smaller ones. A/W in Eq. (2) is not appropriate in terms of screen size variations because the term caused the observed inconsistency. In the following two sections, the authors investigate better formulation of ID.

\section{Fitness evaluation of multiple regression model}

Based on the finding reported in the last section, the authors 1) evaluate the applicability of possible models other than the Fitts' one and 2) make an attempt to improve the definition of ID in the Fitts' model, i.e., Eq. (2). The finding implies that a model in which A and W independently affect the pointing time $t$ may capture the effect of device screen size more appropriately: such a model may be able to represent that A (W) affects more than W (A) where device screen sizes are larger (smaller). For example, a power function model was previously proposed (Kvalseth, 1980).

$$
\begin{gathered}
\mathrm{t}=\mathrm{a} * \mathrm{~A}^{\mathrm{b}} * \mathrm{Wc} \\
\log _{2} \mathrm{t}=\mathrm{a}+\mathrm{b} * \log _{2} \mathrm{~A}+\mathrm{c} * \log _{2} \mathrm{~W}
\end{gathered}
$$

The following model has also been investigated (MacKenzie, 1992).

$$
\mathrm{t}=\mathrm{a}+\mathrm{b} * \log _{2} \mathrm{~A}+\mathrm{c} * \log _{2} \mathrm{~W}
$$

Based on these previous researches, the authors evaluate fitness of multiple regression models in Eqs. (4') and (5) by applying the model to the data collected by user experiments in our research.

By normalizing the data of $t, \log _{2} t, \log _{2} \mathrm{~A}$ and $\log _{2} \mathrm{~W}$ respectively, $\mathrm{a}=0$ and the value of $\mathrm{b}$ can be directly compared with the value of $\mathrm{c}$.

$$
\begin{gathered}
\mathrm{t}^{\prime}=\mathrm{b} * \log _{2} \mathrm{~A}^{\prime}+\mathrm{c} * \log _{2} \mathrm{~W}^{\prime} \\
\log _{2} \mathrm{t}^{\prime}=\mathrm{b} * \log _{2} \mathrm{~A}^{\prime}+\mathrm{c} * \log _{2} \mathrm{~W}^{\prime}
\end{gathered}
$$

In Eqs $\left(5^{\prime}\right)$ and $\left(4^{\prime \prime}\right), t^{\prime}, \log _{2} t^{\prime}, \log _{2} A^{\prime}$ and $\log _{2} W^{\prime}$ are normalized ones respectively. 
Table 6 shows values of $\mathrm{b}$ and $\mathrm{c}$ for the model in Eq. (5') obtained by applying the multiple regression analysis to the data of $\left(\mathrm{t}^{\prime}, \log _{2} \mathrm{~A}^{\prime}, \log _{2} \mathrm{~W}^{\prime}\right)$.

(i) Errors acceptable

\begin{tabular}{|c|c|c|c|c|}
\cline { 2 - 5 } \multicolumn{1}{c|}{} & \multicolumn{2}{c|}{ Device L } & \multicolumn{2}{c|}{ Device S } \\
\cline { 2 - 5 } & Targets L & Targets S & Targets L & Targets S \\
\hline b & 0.13 & 0.23 & 0.10 & 0.04 \\
\hline c & -0.33 & -0.18 & -0.39 & -0.37 \\
\hline
\end{tabular}

(ii) Errors not acceptable

\begin{tabular}{|c|c|c|c|c|}
\cline { 2 - 5 } \multicolumn{1}{c|}{} & \multicolumn{2}{c|}{ Device L } & \multicolumn{2}{c|}{ Device S } \\
\cline { 2 - 5 } \multicolumn{1}{c|}{} & Targets L & Targets S & Targets L & Targets S \\
\hline b & 0.004 & 0.21 & 0.16 & 0.14 \\
\hline
\end{tabular}

Table 6. Values of $b$ and $c$ in Eq. (5')

Table 7 shows values of $\mathrm{b}$ and $\mathrm{c}$ for the model in Eq. (4") obtained by applying the multiple regression analysis to the data of $\left(\log _{2} \mathrm{t}^{\prime}, \log _{2} \mathrm{~A}^{\prime}, \log _{2} \mathrm{~W}^{\prime}\right)$.

(i) Errors acceptable

\begin{tabular}{|c|c|c|c|c|}
\cline { 2 - 5 } \multicolumn{1}{c|}{} & \multicolumn{2}{c|}{ Device L } & \multicolumn{2}{c|}{ Device S } \\
\cline { 2 - 5 } & Targets L & Targets S & Targets L & Targets S \\
\hline b & 0.16 & 0.26 & 0.19 & 0.14 \\
\hline c & -0.30 & -0.15 & -0.33 & -0.32 \\
\hline
\end{tabular}

(ii) Errors not acceptable

\begin{tabular}{|c|c|c|c|c|}
\cline { 2 - 5 } \multicolumn{1}{c|}{} & \multicolumn{2}{c|}{ Device L } & \multicolumn{2}{c|}{ Device S } \\
\cline { 2 - 5 } & Targets L & Targets S & Targets L & Targets S \\
\hline b & 0.07 & 0.27 & 0.22 & 0.25 \\
\hline c & -0.36 & -0.16 & -0.28 & -0.25 \\
\hline
\end{tabular}

Table 7. Values of $b$ and $c$ in Eq. (4')

Tables 6 and 7 revealed the followings.

- Values of $b$ are all positive, and those of $c$ are all negative. Thus, the models by Eqs. $\left(5^{\prime}\right)$ and $\left(4^{\prime \prime}\right)$ appropriately represent that the pointing time becomes larger (smaller) as the target distance $\mathrm{A}$ (the target size $\mathrm{W}$ ) becomes larger.

- $\quad$ For the device $\mathrm{S},|\mathrm{b}|<|\mathrm{c}|$ in both tables so that the target size $\mathrm{W}$ affects the pointing time more than the target distance A. This is consistent with the result reported in the last section.

- $\quad$ For the device $\mathrm{L},|\mathrm{b}|>|\mathrm{c}|$ in some condition combinations (e.g., Table 6(i), the targets S) so that the target distance A affects the pointing time more than the target size $\mathrm{W}$. This is also consistent with the result reported in the last section. However, $|\mathrm{b}|<|\mathrm{c}|$ for the other condition combinations (e.g., Table 6(i), the targets L), which is not consistent with the result. This inconsistency should be further investigated in our future work.

This result shows the multiple regression models by Eqs. $\left(5^{\prime}\right)$ and $\left(4^{\prime \prime}\right)$ well represent the effects of target sizes and distances on the pointing time, especially for the small screen device and partially for the large screen device. 


\section{Improvement in Fitts' law ID formulation}

The authors next investigate an improvement to the definition of ID in the Fitts' model. The advantage of multiple regression models was shown in Section 3, but a drawback of the models is that users' pointing throughput values cannot be calculated. This is because a single index of difficulty is not defined in the case of the multiple regression models.

Our idea for the improvement is to raise A or W depending on the screen size as shown in Eqs. (6) and (7).

$$
\begin{aligned}
& \text { ID }=\log _{2}\left(A^{\alpha} / W+1\right), \quad \alpha>1 \\
& I D=\log _{2}(A / W \beta+1), \quad \beta>1
\end{aligned}
$$

Eq. (6) is employed for larger screen devices and Eq. (7) for smaller ones.

The modified model was applied to the collected data. Appropriate values of $\alpha$ and $\beta$ in Eqs. (6) and (7) are explored by the bisection method so that there was no significant difference between population mean values of throughputs for the targets $S$ and $L$ (i.e., the throughputs were consistent between the two target sets) on the same device.

It is found that the modified model well fits to the data where $a=1.61,1.62$ for the device $L$ and $\beta=1.00,1.15$ for the device $S$ (Tables 8-11): under these values of $\alpha$ and $\beta$, no significant difference is observed between population mean values of throughputs for the targets $S$ and L. Thus, the modified indexes of difficulties by Eqs. (6) and (7) well represent actual pointing difficulties for users so that users' pointing throughputs become consistent on the same device among target design variations (c.f., was inconsistent in the case of traditional ID, Eq. (2)).

\begin{tabular}{|c|c|c|c|}
\cline { 3 - 4 } \multicolumn{2}{c|}{} & \multicolumn{2}{c|}{ Device L } \\
\cline { 3 - 4 } \multicolumn{2}{c|}{} & Targets S & Targets L \\
\hline \multirow{2}{*}{$\begin{array}{c}\text { Errors acceptable } \\
(\alpha=1.62)\end{array}$} & Mean & 12.5 & 12.5 \\
\cline { 2 - 4 } & SD & 2.89 & 1.91 \\
\hline $\begin{array}{c}\text { Errors not acceptable } \\
(\alpha=1.61)\end{array}$ & Mean & 11.9 & 11.9 \\
\cline { 2 - 4 } & SD & 2.60 & 2.25 \\
\hline
\end{tabular}

Table 8. Throughput values (ID by Eq. (6))

\begin{tabular}{|c|c|c|}
\cline { 2 - 3 } \multicolumn{1}{c|}{} & \multicolumn{2}{c|}{ Device L } \\
\cline { 2 - 3 } \multicolumn{1}{c|}{} & Errors acceptable & Errors not acceptable \\
\hline Targets & $\mathrm{t}=-5.75 * 10^{-11}$ & $\mathrm{t}=4.17 * 10^{-12}$ \\
S vs. L & $(\mathrm{a}=1.62)$ & $(\mathrm{a}=1.61)$ \\
\hline
\end{tabular}

Table 9. T-test for throughput (ID by Eq. (6))

\begin{tabular}{|c|c|c|c|}
\cline { 3 - 4 } \multicolumn{2}{c|}{} & \multicolumn{2}{c|}{ Device S } \\
\cline { 3 - 4 } \multicolumn{2}{c|}{} & Targets S & Targets L \\
\hline $\begin{array}{c}\text { Errors acceptable } \\
(\beta=1.00)\end{array}$ & Mean & 5.73 & 5.73 \\
\cline { 2 - 4 } & SD & 1.37 & 1.14 \\
\hline $\begin{array}{c}\text { Errors not acceptable } \\
(\beta=1.15)\end{array}$ & Mean & 4.78 & 4.78 \\
\cline { 2 - 4 } & SD & 1.15 & 1.09 \\
\hline
\end{tabular}

Table 10. Throughput values (ID by Eq. (7)) 


\begin{tabular}{|c|c|c|}
\cline { 2 - 3 } \multicolumn{1}{c|}{} & \multicolumn{2}{c|}{ Device $S$} \\
\cline { 2 - 3 } \multicolumn{1}{c|}{} & Errors acceptable & Errors not acceptable \\
\hline Targets & $\mathrm{t}=-2.68 * 10^{-11}$ & $\mathrm{t}=-3.60 * 10^{-11}$ \\
S vs. L & $(\beta=1.00)$ & $(\beta=1.15)$ \\
\hline
\end{tabular}

Table 11. T-test for throughput (ID by Eq. (7))

This result shows that our idea of ID improvement is effective: the modified ID formulations can capture users' actual pointing difficulties better than the traditional ID. Further evaluations with additional case data will be our future work.

\section{Conclusions}

Index of difficulty formulation in Fitts' law was evaluated from the viewpoint of consistency in widget size\&distance design variations. It was found that ID in Eq. (2) may not appropriately capture actual difficulty: user performances on the same device were not consistent among target designs $(\mathrm{A}, \mathrm{W})$ and $(\mathrm{nA}, \mathrm{nW})$.

Based on this finding, two multiple regression models were evaluated. These models were $\mathrm{t}=\mathrm{F}(\mathrm{A}, \mathrm{W})$ (c.f., $\mathrm{t}=\mathrm{F}(\mathrm{A} / \mathrm{W})$ in the Fitts' model) which predicted the time $\mathrm{t}$ to point a target with the distance $\mathrm{A}$ and the size $\mathrm{W}$. The models are found to be able to appropriately represent that $\mathrm{W}$ affected the index of difficulty more than $\mathrm{A}$ in the case of the small screen touch UI device. The models however did not work so well in the case of the large screen device, which remained to be investigated in a future research.

The authors next tried to improve the Fitts' law ID formulation. Our idea was to raise A or $\mathrm{W}$ depending on the screen size. The modified model was found to fit well to the users' pointing data, which supports our idea.

\section{References}

Fitts, P.M. (1954). The information capacity of the human motor system in controlling the amplitude of movement, Journal of Experimental Psychology, Vol.47, No.6, 381-391

ISO 9241. (2000). Ergonomic requirements for office work with visual display terminals (VDTs) - Part 9: requirements for non-keyboard input devices.

Kvalseth, T. (1980). An alternative to Fitts' law, Bulletin of the Psychonomic Society, Vol.5, 371373

MacKenzie, I.S. (1992). Fitts's law as a research and design tool in human-computer interaction, Human-Computer Interaction, Vol.7, 91-139

MacKenzie, I.S. \& Buxton, W. (1992). Extending Fitts' law to two-dimensional tasks. Proceedings of ACM Conference on Human Factors in Computing Systems (CHI'92), 219226

Oehl, M., Sutter, C. \& Ziefle, M. (2007). Considerations on efficient touch interfaces - how display size influences the performance in an applied pointing task, in Smith, M.J. \& Salvendy, G. (Eds.), Human Interface, Part I, HCII 2007, LNCS 4557, 136-143

Plamondon, R. \& Alimi, A.M. (1997). Speed/accuracy trade-offs in target-directed movements, Behavioral and Brain Sciences, Vol.20, No.2, 279-349 
Ren, X. \& Mizobuchi, S. (2005). Investigating the usability of the stylus pen on handheld devices, Proceedings of The Fourth Annual Workshop on HCI Research in MIS, 30-34 


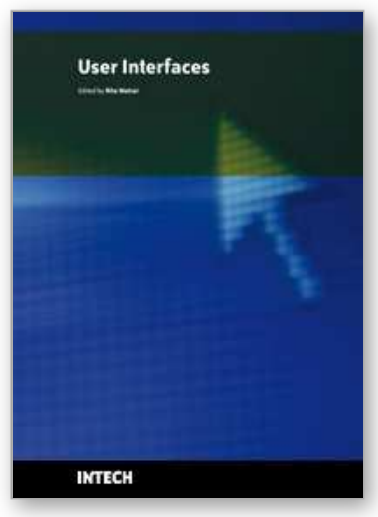

\author{
User Interfaces \\ Edited by Rita Matrai
}

ISBN 978-953-307-084-1

Hard cover, 270 pages

Publisher InTech

Published online 01, May, 2010

Published in print edition May, 2010

Designing user interfaces nowadays is indispensably important. A well-designed user interface promotes users to complete their everyday tasks in a great extent, particularly users with special needs. Numerous guidelines have already been developed for designing user interfaces but because of the technical development, new challenges appear continuously, various ways of information seeking, publication and transmit evolve. Computers and mobile devices have roles in all walks of life such as in a simple search of the web, or using professional applications or in distance communication between hearing impaired people. It is important that users can apply the interface easily and the technical parts do not distract their attention from their work. Proper design of user interface can prevent users from several inconveniences, for which this book is a great help.

\title{
How to reference
}

In order to correctly reference this scholarly work, feel free to copy and paste the following:

Hidehiko Okada and Takayuki Akiba (2010). Fitts' Law Index of Difficulty Evaluated and Extended for Screen Size Variations, User Interfaces, Rita Matrai (Ed.), ISBN: 978-953-307-084-1, InTech, Available from: http://www.intechopen.com/books/user-interfaces/fitts-law-index-of-difficulty-evaluated-and-extended-forscreen-size-variations

\section{INTECH}

open science | open minds

\author{
InTech Europe \\ University Campus STeP Ri \\ Slavka Krautzeka 83/A \\ 51000 Rijeka, Croatia \\ Phone: +385 (51) 770447 \\ Fax: +385 (51) 686166 \\ www.intechopen.com
}

\author{
InTech China \\ Unit 405, Office Block, Hotel Equatorial Shanghai \\ No.65, Yan An Road (West), Shanghai, 200040, China \\ 中国上海市延安西路65号上海国际贵都大饭店办公楼 405 单元 \\ Phone: +86-21-62489820 \\ Fax: +86-21-62489821
}


(C) 2010 The Author(s). Licensee IntechOpen. This chapter is distributed under the terms of the Creative Commons Attribution-NonCommercialShareAlike-3.0 License, which permits use, distribution and reproduction for non-commercial purposes, provided the original is properly cited and derivative works building on this content are distributed under the same license. 